Portland State University

PDXScholar

Civil and Environmental Engineering Faculty

Publications and Presentations

Civil and Environmental Engineering

$10-2018$

\title{
Modeling the Impact of Traffic Conditions and Bicycle Facilities on Cyclists' On-Road Stress Levels
}

\author{
Àlvaro Caviedes \\ Portland State University, caviedes@pdx.edu \\ Miguel A. Figliozzi \\ Portland State University, figliozzi@pdx.edu
}

Follow this and additional works at: https://pdxscholar.library.pdx.edu/cengin_fac

Part of the Civil Engineering Commons, and the Transportation Engineering Commons Let us know how access to this document benefits you.

\section{Citation Details}

Caviedes, Àlvaro and Figliozzi, Miguel A., "Modeling the Impact of Traffic Conditions and Bicycle Facilities on Cyclists' On-Road Stress Levels" (2018). Civil and Environmental Engineering Faculty Publications and Presentations. 456.

https://pdxscholar.library.pdx.edu/cengin_fac/456

This Post-Print is brought to you for free and open access. It has been accepted for inclusion in Civil and Environmental Engineering Faculty Publications and Presentations by an authorized administrator of PDXScholar. Please contact us if we can make this document more accessible: pdxscholar@pdx.edu. 


\title{
Modeling the impact of traffic conditions and bicycle facilities on cyclists' on-road stress levels
}

\author{
Alvaro Caviedes, Graduate Research Assistant \\ Transportation Technology and People (TTP) Lab \\ Department of Civil and Environmental Engineering \\ Portland State University \\ Miguel Figliozzi. PhD, Professor (corresponding author) \\ Transportation Technology and People (TTP) Lab \\ Department of Civil and Environmental Engineering \\ Portland State University \\ figliozzi@pdx.edu
}

\begin{abstract}
Past research efforts have shown that cyclists' safety, stress, and comfort levels greatly affect the routes chosen by cyclists and cycling frequency. Some researchers have tried to categorize cyclists' levels of traffic stress utilizing data that can be directly measured in the field, such as the number of motorized travel lanes, motorized vehicle travel speeds, and type of bicycle infrastructure. This research effort presents a novel approach: real-world, on-road measurements of physiological stress as cyclists travel across different types of bicycle facilities at peak and off-peak traffic times. By matching videos with stressful events, it was possible to observe the circumstances of those stressful events. The stress data was normalized, and the method was carefully validated by a detailed analysis of the stress measurements. Novel statistical results from a multi-subject study quantifies the impact of traffic conditions, intersections, and bicycle facilities on average stress levels.
\end{abstract}

Keywords: Cyclists; Stress; Traffic; Roadway; Bicycle Facilities

\section{Reference}

Caviedes, A., \& Figliozzi, M. (2018). Modeling the impact of traffic conditions and bicycle facilities on cyclists' on-road stress levels. Transportation research part F: traffic psychology and behaviour, 58, 488499. 


\section{Introduction}

Many cities have ambitious plans to increase bicycle mode share in the short and long term. These plans motivated researchers to better understand where and what types of bicycle improvements yield the maximum net benefit in terms of increased ridership, comfort, and safety. Transportation engineers and planners have attempted to estimate cyclists' safety, comfort, stress levels, and level of service using two experimental design approaches: naturalistic and non-naturalistic studies. In a naturalistic approach, the subject is observed or analyzed in his or her natural environment (on-road) while cycling without any significant manipulation or interference. In a non-naturalistic study, the subject is analyzed in an artificial setting or provides data executing an activity that is not cycling-for example, in a laboratory or through a web-survey.

Most non-naturalistic studies have used surveys, photos, and/or films to understand cyclists' preferences. For example, the subjects evaluate different environments or facilities by looking at pictures of intersections and/or corridors. Non-naturalistic studies are useful to investigate cyclists' perceived comfort and preferences for various types of bicycle facilities. Nonetheless, this approach may result in bias due to the subjectivity of the metrics and the nature of stated versus revealed (or measured) preferences or responses. Surveys and laboratory settings do not always reflect the psychological and physiological responses that occur in a natural setting. Picard and Healy (2001) suggest that when measuring human emotions and perceptions, the researcher has to be aware that questions related to a subject's personal feelings can produce answers which vary "according to her awareness of her feelings, her comfort in talking about feelings, her rapport with the administrator of the experiment and more."

Naturalistic studies are designed to collect data by investigating the subject in his or her natural riding environment without any significant interference. Using video, Johnson et al. (2001) found that the main variables associated with red light infringement are a function of cyclists' safety perceptions of opposing traffic volumes. Chuang et al. (2013) collected field data using a bicycle equipped with various sensors and cameras. The authors found that cyclists' behavior changes with proximity to motorized vehicles. They also found that a demarcated bike lane generates a higher distance between the cyclist and the motor vehicle than riding on the right of the motorized vehicle lane without demarcation; more separation from motorized vehicles is perceived positively by cyclists (Carter, Hunter, Zegeer, \& Stewart, 2007). The Bicycle Level of Service (BLOS) of the 2010 Highway Capacity Manual (HCM) measures the performance of bicycle users and facilities using data directly collected in the field (Transportation Research Board, 2010); however, this methodology is not based on naturalistic data as defined in this paper; users were asked to rate different types of facilities after watching video clips filmed from a bicycle. Alternately, using revealed GPS data from onroad, real-world bicycle trips, Harvey et al. (2008) found that cyclists are willing to travel longer distances to their destinations in order to achieve high levels of safety and comfort. A limitation of the cited naturalistic studies is the lack of consideration of stress measurements while the subject is cycling - videos and bicycle sensors cannot measure the level of stress that a cyclist is experiencing while making a route decision or simply biking.

Summarizing, naturalistic and non-naturalistic studies have pros and cons and ideally complement each other. This research is a multi-subject research study linking real-world, on-road cyclists' stress measurements with roadway characteristics. Some researchers have previously used the term stress to characterize bicycle-riding conditions. For example, bicycle level of stress (BLS) has been described as a function of safety levels and physical/mental effort and age (Sorton \& Walsh, 1994), while level of traffic stress (LTS) is a function of traffic/geometric variables and the different user groups' characteristic within the population (Mekuria, Furth, \& Nixon, 2012). Despite their names, neither BLS nor LTS is based on real-world, on-road stress measurements. BLS and LTS are relatively intuitive and simple to apply, but they are not supported by realworld, on-road empirical evidence. This research is the first step to filling this research gap. 
The key research questions of this study are the following: (a) what is the impact of traffic conditions (peak vs. off-peak) on cyclists' stress levels, and (b) what is the impact of different facility types (bike lane, shared road, dedicated path) on cyclists' stress levels?

\section{Stress Background}

The term stress can be defined as "the non-specific mix of physiological and psychological responses of the body to any demand of change" (Seyle, 1956). It is a reaction from a calm state to an excited state for the purpose of being alert during a situation in which the subject feels threatened or attacked. Stressful events, such as biking too close to motorized traffic, cause dynamic changes in the autonomic nervous system (ANS); more specifically, stressful events cause an increase in sympathetic nervous system (SNS) activity (Wilfrid \& McLachlan, 1992) and a decrease in parasympathetic nervous system (PNS) activity, which is evidenced by changes in heart rate, blood pressure, breathing rate, and galvanic skin response (GSR) (Sharma \& Gedeon, 2012).

For nearly four decades, psychologists have studied stress levels by collecting physiological data. Various studies have found a definitive association between specific human emotions and physiological responses such as heart rate, skin conductance, blood pressure, breath rate, etc. (Boucsein, 2012). Physiological responses can also be used to predict emotional state. Friedlund and Izard (1983) were the first to recognize emotions from physiological responses, attaining rates of 38-51\% accuracy using electromyogram signals (an electromyogram, or EMG, measures the electrical activity of muscles at rest and during contraction). It is possible to differentiate states of anger and fear $(\mathrm{Ax}, 1953)$ and states of conflict and non-conflict using GSR and heart rates (Kahneman, 1973). Ekman et al. (1983) found evidence for distinctive patterns of autonomic nervous (ANS) activity for anger, disgust, and possibly sadness. Helander (1978) found that GSR was the best predictor to measure the impact of stressful events on a subject. Labbé et al. (2007) found that when an individual is under stress, skin conductance increases due to an increase in sweat activity. Physiological responses have the potential to represent internal human states influenced by cognitive and social variables (Picard, Vyzas, \& Healy, 2001); some internal human emotional responses can be measured using noninvasive methods (Lanzetta \& Orr, 1986).

GSR-based studies have been successfully employed for many years in the psychological field to recognize and associate emotions and behaviors to physiological responses. In previous studies, GSR yielded the most accurate data for measuring stress in human subjects compared to surveys or questionnaires (subject to cognitive biases). Skin conductance (also known as GSR) is one of the most robust, non-invasive physiological measures of ANS and electrodermal activity to investigate stress (Healy J. , 2000) (Picard, Vyzas, \& Healy, 2001) (Lisetti \& Nasoz, 2004) (Dawson, Schell, \& Filion, 2007). GSR sensors measure the changes in the conductance of skin caused by ionic sweat secretion (which is related to stressful events). "The resistance of the skin is usually large; however, momentary changes in the level of the sweat gland activity causes changes in resistance that can be measured by passing a small electrical current across two electrodes placed on the surface of the skin" (Healy J., 2000). Studies in other fields, mainly psychology and medicine, have demonstrated that emotional arousal (such as stress) leads to an increase in skin conductance (Healy \& Picard, 2005) (Seyle, 1956) (Labbé, Schmidt, \& Babin, 2007).

Ekman et al. (1983) and Lanzetta and Orr (1986) found that emotions like fear and disgust produce a larger increase in GSR than happiness. Pecchinenda and Smith (1996) measured GSR in subjects at the beginning of a problem-solving task and found that the signal increased significantly from the baseline; then, when the subjects were more comfortable and confident with the situation, the signals started to decrease again as expected. Lisetti and Nasoz (2004) used movie clips and difficult mathematics questions to elicit various emotions, finding that GSR signals could explain fear. Using GSR measures, Labbé et al. (2007) found that listening to classical music or self-selected relaxing music after being exposed to a stressor leads to reduced anxiety and alertness when compared to individuals that sit in silence or listen to heavy metal music. 
Helander (1978) found that GSR is a good predictor for driver stress in events such as encountering a cyclist, pedestrian, or another car merging in the street. Healy and Picard (2005) found that GSR sensors can be used to obtain signals processed by on-board car computers to give dynamic, reliable measures of drivers' internal states while they are driving in real, on-road environments.

A recent, innovative study that is closely related to this research is the cycle BOOM project (Jones et al., 2016) that involved 240 participants in several UK cities. The aim of this study was to measure the impact of cycling on both physical and mental health, and a part of the study utilized GSR measurements. The GSR data showed that in many instances, high levels of stress corresponded with video observations and rider descriptions of conflicts with pedestrians. The researchers also found that GSR baselines vary greatly by subject, and to overcome this issue, the participant data was allocated in three bands of different colors: red, yellow and green for high, medium, and low measurements respectively. Maps were created by aggregating the bands in 50-meter-long segments. The locations where participants showed high GSR values corresponded to intersections where participants expressed that they had safety concerns. The data seemed to show that participants were more relaxed in traffic-free environments with no pedestrian conflicts. This study also indicates that the data requires further analysis.

There are some key differences between this research and the cycle BOOM project: (a) In this research the data was normalized following the methodology used in most peer-reviewed GSR studies (see equation, Section 4). In the cycle BOOM project, the data was not normalized. Normalization is necessary to address the baseline problem detected by earlier GSR research. (b) This research was designed to test different cycling facilities and to perform a careful statistical analysis taking into account subject variability (see the result of the random effects model in Section 8). (c) There was careful and thorough validation and data collection procedure that involved novel measurements (power, speed, location, etc.) but also baseline data collection periods before and after the on-road measurements. (d) The data granularity for location, time, and GSR is finer in this study.

Summarizing, measures of GSR have been used to study bicycle transportation (Jones et al., 2016) and measure stress levels in automobile drivers (Helander, 1978). To the best of the authors' knowledge, no study has utilized normalized GSR data to study the impact of different road facilities and traffic conditions on cyclists' stress levels. In addition, previous transportation studies have not utilized random effects models to better account for subject variability.

\section{Data Collection}

This research was designed to monitor cyclists' physiological reactions (GSR) under real-world cycling situations, such as different types of bicycle facilities and varying traffic volumes. Based on the published findings previously discussed, GSR data collected from cyclists is assumed to be directly related to stress levels. In Section 6, it is shown that after being normalized, the collected data is not affected by physical exertion (cycling power) or environmental conditions (temperature).

A pilot study was implemented during weekdays in July of 2015 to evaluate the sensors and study design. From a subject not included in the final data collection, seven days of data were collected during peak and off-peak hours in downtown Portland, Oregon. Comparing the stress measures with the videos recorded, it was observed that the GSR sensor captured stress responses to traffic conflicts and roadway conditions. For the pilot study, the findings suggested that there is a significant difference between stress levels at different times of the day. Stress levels during peak-hour were on average 1.75 times higher than off-peak levels. Signalized intersections were hotspots for stress, too; at intersections, stress levels were 1.7 times higher during peak hours than at the segments connecting intersections at off-peak periods (p-value < 0.05). Finally, the findings suggested that there is a significant difference in stress levels between different facilities. On 
average, biking on physically segregated facilities (off-street) caused less stress than biking on shared roadways (p-value $<0.05$ ).

The final data collection took place during seven weekdays in October, 2015. This time of the year was selected because temperatures in Portland are pleasant to ride, with high-low temperatures in the 16-8 centigrade range (61-46 in Fahrenheit). GSR and Global Positioning System (GPS) data were collected for the same route utilized in the pilot study. The route was composed of segments with two distinct types of bicycle facilities: on-street (shared roadway with mixed traffic conditions, $1.49 \mathrm{~km}$; urban streets with bike lanes, $2.27 \mathrm{~km}$ ), and off-street (multi-use path, $4.94 \mathrm{~km}$ ). A multi-use path is a lane physically separated from motorized traffic and is mainly used by pedestrians and cyclists. Data was collected at morning peak and offpeak hours. GSR signals were also collected for five minutes before and after the ride to establish a baseline state for the subjects. We used these baseline periods as a control for potential measurement problems associated with sweat or other uncontrolled data collection variables.

Five healthy subjects different from the pilot study subjects who were between 22 and 33 years old with different levels of cycling experience took part in this study. The subjects had to follow the same procedure and ride the same route. To participate in the experiment, the subjects were required to consent to cycle defensively and to obey all traffic signals during the rides. The data collection procedure and study were approved by the university research internal review board (IRB). Before the first ride, the subjects reviewed the study route with the researcher and had the opportunity to ask questions. The subjects took between 24 and 27 minutes to complete each ride, depending on the day of the week and time of day-peak 8:00 AM vs. off-peak 12:00 PM. Approximately seven hours of GSR, video, and GPS data were recorded. The data collection was balanced; each subject had to ride the same bicycle and same route in the same conditions (peak and off-peak traffic).

GSR signals were recorded using the Shimmer3 GSR+ sensor, which has been validated for accuracy by many research studies in fields ranging from sports medicine to vehicular and environmental monitoring (see http://www.shimmersensing.com/support/publications/). This sensor is a noninvasive device consisting of one monitor and two electrodes. The monitor was mounted on the arm of the subject's nondominant hand, and the electrodes were attached to two fingers. Normally, electrodes are attached to the middle of the three segments of the first and middle finger on the palm of the nondominant hand; however, this placement would cause problematic contacts that could interfere with and distort signals while riding a bicycle. Fowles et al. (1981) found that GSR signals are not affected by the contact area as long as areas of the skin with different potentials are not connected together. They suggest placing the electrodes on any site of the thenar and hypothenar eminences of the nondominant hand. Factors such as hand washing were taken into account before attaching the electrodes (Picard, Vyzas, \& Healy, 2001). GSR data was sampled at $50 \mathrm{~Hz}$.

The bicycle (Figure 1) was purchased and customized for this research. It was selected based on its suitability for various groups of cyclists. It has a lowered top tube, handlebars for upright riding, and is mounted with a power meter to measure the subjects' cycling performance (power, torque, wheel speed and distance, and crank cadence utilizing a PowerTap G3 32H Shimano Hub). Temperature data was collected from the publicly available data sources and thermometers. Since the power meter collects data on power, we calculated energy by simply integrating the power curve over the desired time. To validate the datasets, we compared GPS speeds with power meter speeds. Utilizing 5-second intervals no outliers were observed and the linear correlation was approximately 0.99 . GSR raw data was aggregated by half a second to synchronize it with the GPS data. GPS data and the output from the power meter were collected from an app we developed for this study. The app was uploaded into a phone (Samsung Galaxy S5) mounted on the bicycle handlebar. GSR data was also collected by using the same smartphone so the data shared the same time clock. Finally, two GoPro cameras were mounted on the subjects' helmet to record a 360-degree view around the cyclist. We recorded the time that was on the phone at the beginning of the ride to synchronize the time of the data collected and the video so that we could align video data with the GPS and stress data. 


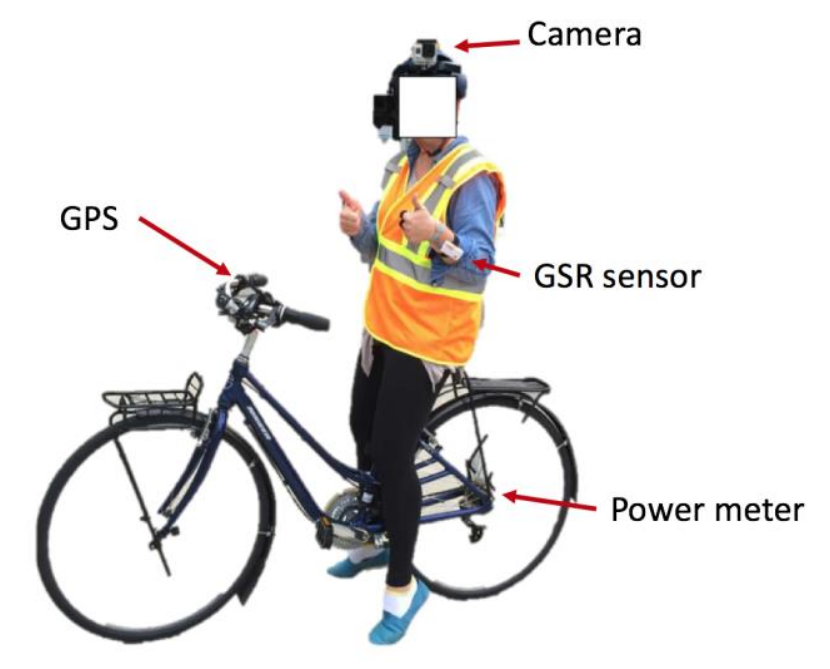

Figure 1. Bicycle and Sensors.

\section{GSR signal processing}

GSR data typically shows high frequency fluctuations; hence, data was smoothed by a moving average with a size of five seconds, as done in previous studies (Picard, Vyzas, \& Healy, 2001). Then, GSR was normalized following the methodology used in most GSR studies, such as Healy (2000) (see equation 1). These features were chosen to represent the autonomic level during the emotion episode and the change in autonomic activation over the course of the episode (Healy, 2000).

$g_{n_{i}}=\left(g_{i}-\bar{g}_{b}\right) / \bar{g}_{b}$

where:

$g_{n_{i}}=$ GSR-normalized signal

$g_{i}=$ GSR observation $i$ utilizing a 5-second moving average

$\bar{g}_{b}=$ Subject GSR 5-minute mean before starting the ride.

A benefit of utilizing equation (1) is that observations during the ride are relative to the state of the individual immediately before taking the ride. Figure 2 shows typical features of a GSR signal: latency, amplitude, rise time, and half recovery time (Healy, 2000). GSR onset occurs a few seconds after the stimulus is experienced (latency). Some studies suggest that GSR within a time window of 1-5 seconds are attributed to stimulus (Picard, Vyzas, \& Healy, 2001) (Lisetti \& Nasoz, 2004) (Prokasy \& Raskin, 1973). The amplitude $\left(g_{i}\right)$ of the signal is the physiological response of the subject to the stimulus. It decreases until the subject achieves its normal physiological state as long as no more stimuli are experienced. After identifying the amplitude, we recorded the duration of response (rise). 


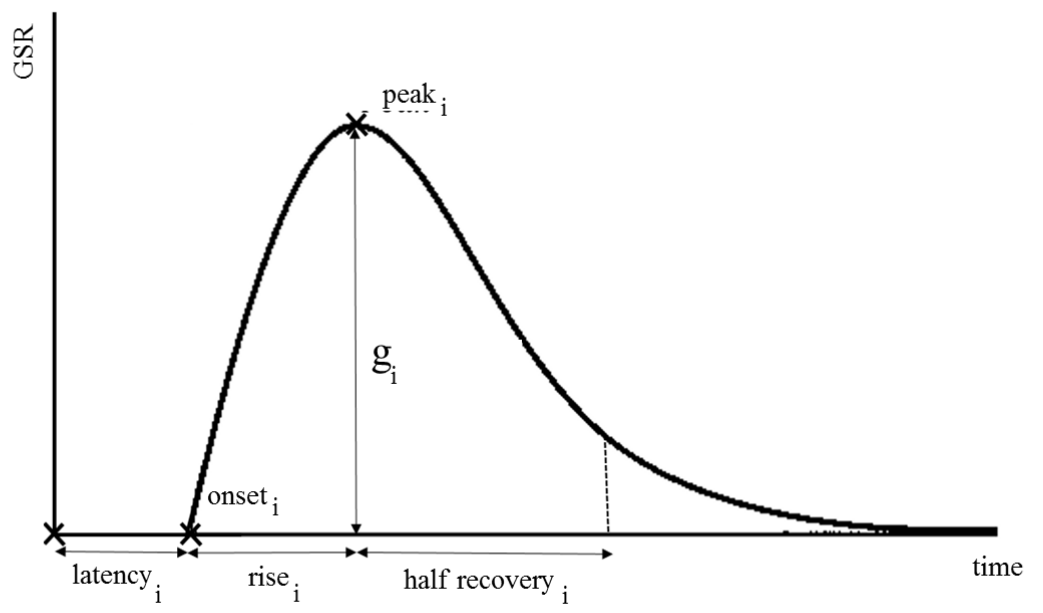

Figure 2. GSR signal features.

When the GSR level increases or peaks in a short period of time, this is usually a physiological response to a specific stressful event (Healy J. , 2000). The stress responses (GSR), or peaks, were identified by first detecting changes in slope (e.g., from positive to negative) through the response ration and then finding local minimum and local maximum preceding and following that point, respectively. As in previous studies, the response was hypothesized to be the value that better represents the subjects' response to a specific event. The response was calculated utilizing equation (2).

$r_{j}=g_{n_{j}} /$ rise $_{j}$

where:

$r_{j}=$ response for event or peak $j$

$g_{n_{j}}=$ GSR-normalized signal around event or peak $j$

rise $_{j}=$ duration of response for observation $j$

Equation (2) is attempting to measure the slope of the response. Previous research has shown that steeper slopes are associated to more stressful events. Statistics for the GSR-normalized signals $g_{n_{i}}$ and for the response $r_{j}$ were used to investigate how various characteristics of the trip affect stress levels and what events are associated to steep responses respectively.

\section{Results Utilizing Data Visualization}

To visualize the GSR data and the impact of bicycle facilities, Figure 3 shows the locations with the highest response, $r_{j}$. This map was made by standardizing responses across subjects and then identifying clusters of stressful events. To standardize responses, we identified the events above two standard deviations from the mean (we hypothesized these were the most stressful events) for each subject. After identifying these events, we merged this information to create a hotspot map (since video and GSR data shared the same time clock, we could readily identify these stressful points). Figure 3 also shows photographs of the facilities encountered at locations with relatively high or low stress levels. The cyclist traveled the route counterclockwise.

Figure 3 shows that the highest level of stress is found in location/photo 4 . Location 4 is an intersection with the highest volume of cross traffic along the route and two merging/right turn lanes that intersected the 
subjects' route. To cross this intersection with state route 99 West Pacific Highway, the subjects had to wait for two green lights. In addition, there is high traffic volume. As shown in Figure 4, vehicles can turn right on the ramp toward the Morrison bridge first and a few feet up toward SW Morrison street. The cyclists followed the trajectory marked by the red arrows toward SW Morrison street. Cyclists are traveling with high-volume through-traffic on the left, but they are also competing with the trajectory of right turning vehicles as they try to turn toward SW Morrison street.
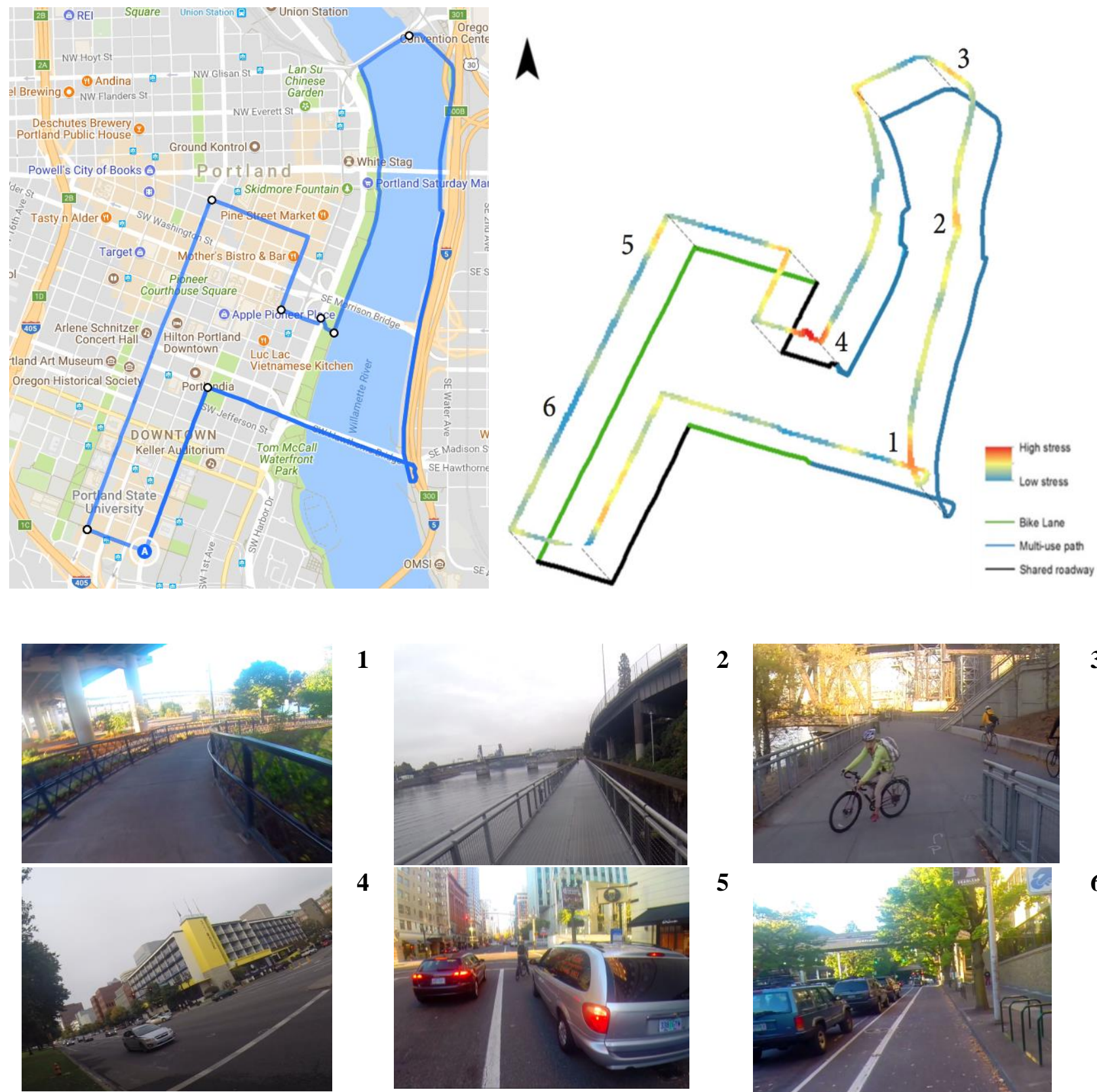

1

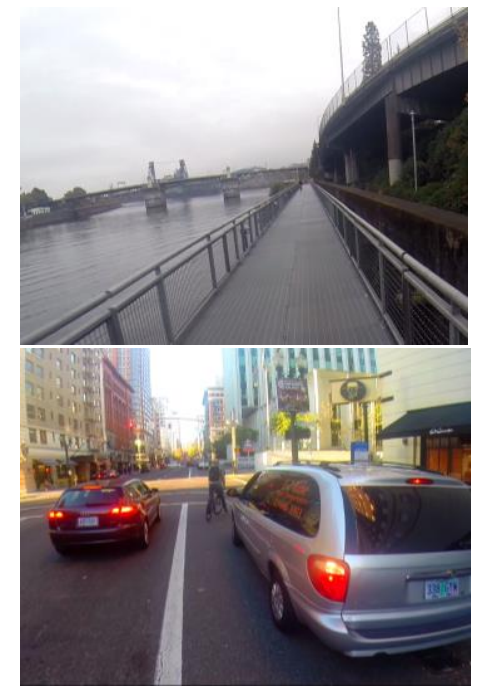

2

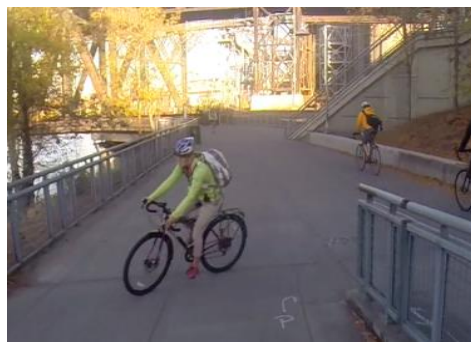

5

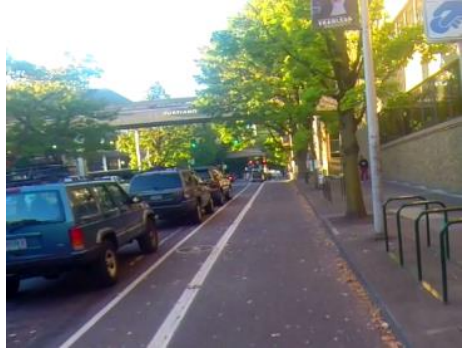

Figure 3. Stress level (upper level) and facility type (lower level). Photographs of locations 1-6. 


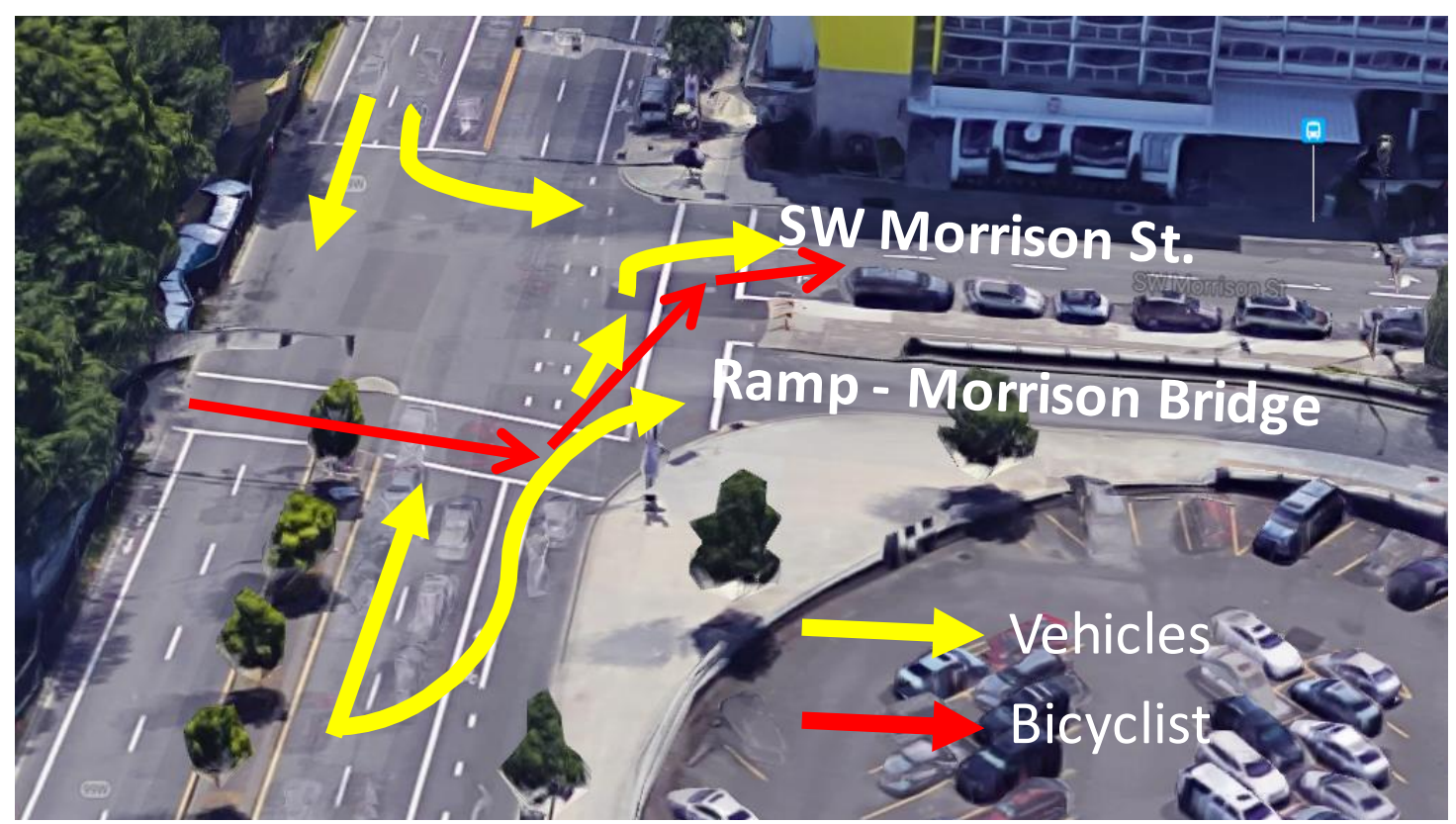

Figure 4. Most stressful location and path (Location 4).

Another stressful location was the bike lane segment (non-protected) shown by Location 5; there is a drastic decrease in stress levels when the cyclists use the protected or buffered bike lane (Location 6). It is worth mentioning that, in general, travel along bicycle lanes near intersections or close to parked vehicles is associated with high stress levels. A different response is observed along the protected or buffered bicycle lane. At Location 6, the protected one-way bike lane has a parking buffer that provides a physical barrier between the cyclist and the vehicle lane.

Along facilities completely separated from traffic, the subjects did not experience significant levels of stress. In particular, the lowest stress levels were observed before arriving at Location 3. This was a segment with a relatively low number of interactions with pedestrians and other cyclists. At other locations along off-street facilities, it was possible to observe more stress (see the off-street segments connecting Locations 1, 2, and 3). At the end of the Hawthorne Bridge, Location 1, there is a steep and very sharp ramp that connects the offstreet path along the bridge with the off-street bicycle path that runs below the bridge and along the river. At Location 2, there is a bicycle-pedestrian bridge that is narrower than the regular path with a rough, uncomfortable surface. At Location 3, two bicycle paths intersect, and there is a high level of conflict with pedestrians and other cyclists that are turning, merging, or diverging.

From the collected videos, it was possible to observe that the occurrences of stress peaks were related to four factors: (i) moving motorized vehicles too close to a bicycle lane or approaching the cyclist, (ii) motorized vehicles invading the bicycle lane, for example a bus crossing in front of the cyclist to serve passengers at a bus stop, (iii) vehicles turning left or right at intersections, (iv) pedestrians invading the bicycle lane (i.e., at the buffered lane) or with a trajectory or unexpected movement that conflicts with the bicycle trajectory in the off-street facilities. In general, any event that was unexpected or potentially dangerous registered high response in the GSR data. The majority of the stressful events took place when the cyclists were utilizing a bike lane or at intersections during peak traffic (more vehicles, more conflicts). The results are qualitatively similar to the results from the cycle BOOM project (Jones et al., 2016). 


\section{Validation of the data collection method}

It was mentioned in the literature review that GSR sensors measure the changes in the conductance of skin caused by ionic sweat secretion (which is related to stressful events). When bicycling, the subject is likely to sweat due to exertion. This begs the questions, are GSR measurements useful to measure on-road cycling stress?

Several steps were taken to ensure the data collection quality and validate the results: (a) to minimize sweating, data collection was conducted in October (a month with cool and pleasant cycling temperatures in Portland) and data collection days had temperatures in the 10-15 centigrades range. (b) Detailed measurements of the cyclists were taken for 5 minutes before and after the on-road data collection to establish before and after baseline states for the subjects. (c) After the data collection, subjects were asked to locate on a map the places where they felt stressed or uncomfortable. (d) GSR responses and bicycle measurements (power, speed, and torque) were plotted and analyzed.

The GSR signals collected for five minutes before and after the ride were used to establish a baseline state for the subjects. The reader is reminded that GSR readings fluctuate even if the subject is in repose, it is impossible to reduce the measurements to zero. Hence, the changes and the frequency of the changes are measured. In the baseline periods the cyclist was riding in an off-street quiet area with very few pedestrians and interactions. The purpose of these baseline periods was to validate the stress measurements and to control for potential measurement problems associated with sweat or other uncontrolled data collection variables during the ride (for example, an electrode may move or loosen up). In all cases the baseline states showed a remarkable decrease; in all cases, utilizing paired t-tests, the baseline mean responses were significantly lower than in the less stressful segment of the ride.

After the ride, the riders were asked to locate the places where they felt stressed or uncomfortable on a map. The riders consistently mentioned higher discomfort during peak-traffic conditions, such as the location shown in Figure 4, and when parked cars and/or vehicles approached their trajectory. There was an overall agreement between riders' comments and the map presented in the previous section (riders' comments were taken right after the ride and before the data was processed, i.e. riders' comments were not influenced by the stress map).

GSR measurements were plotted against different variables that may affect sweating, like power, energy, speed, and temperature. Figure 5 shows six plots displaying the relationship between response $\left(r_{j}\right)$ and power (Watt) for the pilot study and the 5 subjects. The graphs and the very low correlations suggest that there is no clear relationship between response $\left(r_{j}\right)$ and power (Watt) — similar graphs can be observed for the other variables: energy, speed, and temperature. A statistical test for the correlation failed to reject the null hypothesis stating that the correlation coefficient was equal to zero (at a 0.01 significance level).

Likely explanations for the lack of relationships are that temperatures were cool, the route was mostly in flat terrain, and that the response is calculated from the local rises and slopes in the GSR signal and not the absolute value of the signal. By normalizing the responses, the current level of sweat is removed from the observation and only the relative changes are utilized to measure stress. It is not surprising that when power, energy, speed, and temperature are included in the statistical models, they are not significant as detailed in a later section. It is important to highlight that the raw GSR measurement - not normalized utilizing equation (1) — shows a slightly higher level of correlation (yet not significant) with power and energy variables, as expected. 

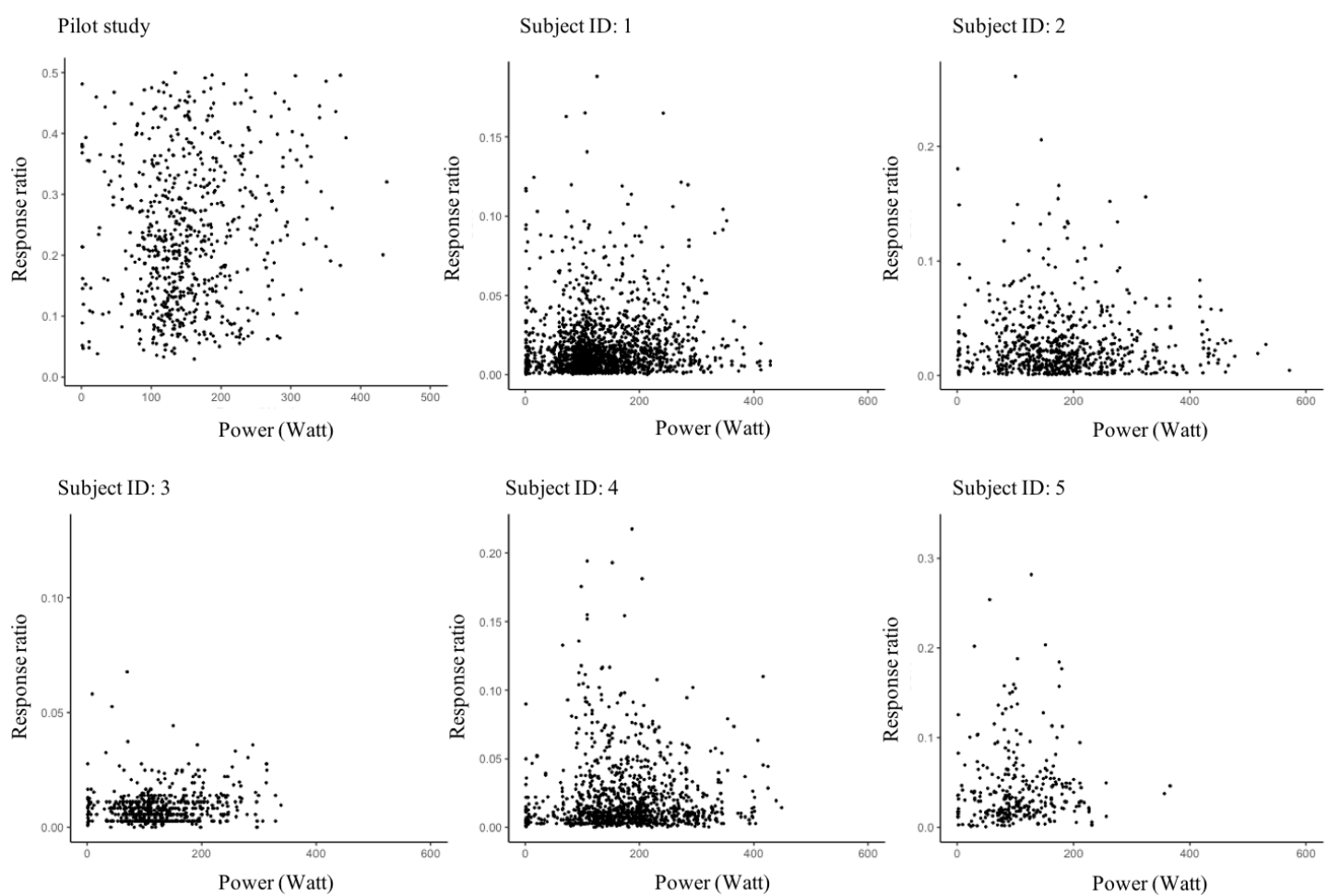

Figure 5. Response and power relationship.

\section{Exploratory results utilizing paired t-tests}

A paired t-test is more appropriate than an independent $t$-test because there is an intrinsic relationship between the observations collected in the two samples (same subjects). Hence, to compare responses by time of day (peak vs. off-peak) and type of facility or segment type (intersections vs. segment), paired t-tests were utilized. The paired t-tests compared the average of responses. Table 1 presents a summary of the descriptive statistics of the responses for different conditions. The number of observations show the number of points measured by the GSR sensor (after estimating the moving average) for the 5 subjects, under different conditions (time of day, segment type, and type of facility).

Table 1. Descriptive statistics for the subjects' responses.

\begin{tabular}{lccccc} 
& Observations & $\mathbf{1}^{\text {st }} \mathbf{Q t}$ & Mean & Median & $\mathbf{3}^{\text {rd }} \mathbf{Q t}$ \\
\hline Time of day & 3,984 & 0.005 & 0.021 & 0.012 & 0.026 \\
$\quad$ Peak hour & 3,115 & 0.005 & 0.018 & 0.011 & 0.021 \\
$\quad$ Off-peak hour & 711 & 0.005 & 0.022 & 0.013 & 0.029 \\
\hline $\begin{array}{l}\text { Segment type } \\
\text { Intersection }\end{array}$ & 6,388 & 0.011 & 0.019 & 0.011 & 0.022 \\
$\quad$ Segment of the road & & & & & \\
\hline Type of facility & 4,259 & 0.005 & 0.018 & 0.010 & 0.021 \\
$\quad$ Off-street & 2,840 & 0.006 & 0.026 & 0.012 & 0.025 \\
$\quad$ On-street & 7,099 & & & & \\
\hline Total & & & & & \\
\hline
\end{tabular}


For this analysis, we filtered the data by peak and off-peak or by type of facility. After doing this, we estimated the averages and then compared them using a paired student t-test. The route contained 44 signalized intersections and only 2 non-signalized intersections. A buffer of 10 meters around each intersection was used to select GSR signals that occurred at intersections. Type of facility refers to on-street and off-street bicycle facilities.

\subsection{Peak traffic}

The first hypothesis stated that the subjects experienced the same average stress level for the two periods of the day, peak and off-peak traffic, with $H_{0}=\mu_{\text {peak }}-\mu_{\text {off-peak }}=0$ and $H_{a}=\mu_{\text {peak }}-\mu_{\text {off-peak }}>0$. The data indicates that the average stress level during peak hours is higher than at off-peak hours, with a p-value < 0.01 .

The average response during peak hours is $17 \%$ higher than the average response during off-peak hours. Stress levels differed across various facilities when the subject was riding at peak and off-peak hours. Morning peak hours resulted in more stressful rides for cyclists due to higher traffic volumes. Stressful events tended to occur mostly at intersections or locations where the subject was riding in a shared roadway or in a bike lane (on-street) adjacent to mixed traffic.

\subsection{Intersections}

The second hypothesis stated that the subjects experienced the same average stress level at intersections and in segments between intersections; in this case $H_{0}=\mu_{\text {intersection }}-\mu_{\text {segment }}=0$ and $H_{a}=\mu_{\text {intersection }}-$ $\mu_{\text {segment }}>0$.

Regardless of the time of day, most of the stressful events were located at intersections where there are more conflicts and where cyclists must pay more attention to the changing environment. Average stress levels were higher at intersections than along segments of the route, with a significant $p$-value $<0.01$. At the peak hour, the trend is similar; signalized intersections were more stressful than segments of the route. At off-peak hours, response in route segments and signalized intersections were not statistically different utilizing a p-value $=$ 0.05 .

\subsection{Facility type}

The third hypothesis stated that the subjects experienced the same average stress level when riding along onstreet facilities and off-street facilities, $H_{0}=\mu_{\text {on-street }}-\mu_{\text {off-street }}=0$ and $H_{a}=\mu_{\text {on-street }}-$ $\mu_{\text {off-street }}>0$.

Intersections were excluded from the on-street facility data, because off-street facilities do not have intersections. Even after removing intersections, the data indicate that subjects experienced higher stress levels along on-street facilities regardless of the time of the day ( $\mathrm{p}$-value $<0.01$ ).

\subsection{Events by distance}

An analysis correcting by distance traveled reveals similar differences. Table 2 shows the breakdown of top events (highest responses) per kilometer and by type of facility (columns two to four). On-the-road facilities (bike lane and shared path) show a higher frequency of stressful responses than multipath (off-road). Regarding peak and off-peak hour conditions, an analysis of events per distance is not possible since the distance (route) was the same. However, it is possible to show the corresponding percentage of events. The peak hour time period contains over $72 \%$ of the most stressful events per rider. 
It is important to clarify that a multipath environment or even a dedicated bicycle path will never be "eventfree". According to the definition of stress presented earlier, "stress is a reaction from a calm state to an excited state for the purpose of being alert...", hence, the presence of an approaching cyclist or even a squirrel (or other animal) will likely trigger a stress response that is detected by GSR. As discussed in this section and the following, the question whether frequencies and/or mean responses change as a function of facility type or traffic conditions.

Table 2. Descriptive statistics for the subjects' responses correcting by distance.

\begin{tabular}{lcccc} 
& & \multicolumn{3}{c}{ Events per km } \\
\cline { 2 - 5 } & \% Peak hour & Multipath & $\begin{array}{c}\text { Shared } \\
\text { Road }\end{array}$ & Bike Lane \\
\hline Top 300 events & $72.4 \%$ & 30.4 & 34.9 & 39.2 \\
Top 200 events & $73.3 \%$ & 18.7 & 23.1 & 28.5 \\
Top 100 events & $73.9 \%$ & 9.5 & 10.8 & 14.1 \\
\hline
\end{tabular}

\section{Results utilizing a random effects model}

To allow for individual effects, a random-effects model is utilized in this section. The model allows for variability across subjects. This is important because subjects do not have the same response or GSR peak even in a controlled environment and presented with the same stressful event. Normalizing the data as indicated in Section 4 is also useful to remove serial correlation. Serial correlation tests among the dependent variable and lagged dependent variable (lags up to a minute) showed no serial correlation. The residuals (at different lags) of the random effects model did not show correlation either.

All potential variables were included in the initial model and insignificant variables were removed one at the time (based on high p-values) until only significant variables at the 0.05 level remained in the model. During this process there were no changes in the signs of the significant variables. The estimated coefficients for the significant variables were stable too. The results were very stable and the sign of the variables did not change when running individual and pooled (all variables) models. The variables power, energy, speed, and temperature were never significant.

In all models, peak-hour and signalized intersections were always positive as expected; peak-hour was always highly significant. Also as expected, the random effect was always significant, which indicates significant variability across subjects. Among the facility variables, bike lane and multi-use path (off-street) were significant at a p-value $<0.05$; the signs were positive and negative respectively, which was expected.

The final model is presented in Table 3. Taking into account the impact of the peak hour and intersections, biking in mixed traffic conditions is a baseline to analyze the impact of the variable bike lane and multi-use (separated facility) path. The results indicate that bicycling along a bicycle lane is more stressful than cycling utilizing a whole traffic lane. One potential explanation is that in a bicycle lane, cyclists are confined to a narrow lane and sandwiched between parked vehicles on the right and motorized vehicles on the left. The video showed that stress levels tended to increase when there was parking activity or when vehicles passed close to the cyclist. In addition, there may be a factor related to the city and cycling culture. The League of American Bicyclists has classified Portland as a "Platinum" city. In Portland, drivers are used to high 
numbers of bicycles sharing the road, and most drivers tend to be very courteous and provide a comfortable distance between vehicles and bicycles (as was the case in this study). Finally, it is important to mention that the speed limit in the Portland downtown area is mostly 20 miles per hour. In this low-speed environment, sharing the lane with cars is likely to be less stressful than being confined to a bike lane.

Finally, the peak hour is significant and estimated with a value around 3.40 in all models. Signalized intersections w not significant in the pooled model. These results are a useful starting point, but more data collection efforts in other cities and cycling environments are necessary before the results of these models can be generalized.

Table 3. Random effect models results (dependent variable GSR response).

\begin{tabular}{lll}
\hline Fixed effects & \\
Intercept & $2.09 \mathrm{E}-02$ & $(*)$ \\
Peak hour (dummy) & $3.43 \mathrm{E}-03$ & $(* *)$ \\
Signalized intersection (dummy) & $1.61 \mathrm{E}-03$ & \\
& & $(* * *)$ \\
Facility Type & $2.43 \mathrm{E}-03$ & $(* * *)$ \\
Bike Lane & $-4.74 \mathrm{E}-03$ & \\
$\begin{array}{l}\text { Multi-use path } \\
\text { (shared roadway - baseline) }\end{array}$ & \\
& & $(*)$ \\
\hline Random Effects & Variance & \\
Subject & $1.29 \mathrm{E}-04$ & \\
\hline
\end{tabular}

Number of observations: $7099-(*)$ p-value $<0.05,(* * *)$ p-value $<0.001$

\section{Discussion and Conclusions}

The GSR-based data collection approach presented in this research allows the researcher to collect data that measures how the cyclists feel second-by-second without interfering with cyclists' behavior or asking them about their perception of the trip (which can be biased by a recent event or by how the questions are framed/sequenced). Rather than having a subjective average stress measure for the entire travel route, it is possible to precisely identify the places and/or situations where the cyclist felt more stressed.

It is key to normalize the GSR data before attempting any statistical modeling or data analysis. It is also important to validate the data by measuring control baseline periods before and after the data collection and/or utilizing post-ride questionnaires. Researcher should carefully analyze whether power, speed, or temperature are affecting the results. The plots of averaged, normalized results should indicate areas where cyclists' have also noticed discomfort.

A goal of this research was to analyze the suitability of GSR data to study cyclists' stress levels, because this is the first study that has analyzed on-road GSR data from multiple subjects. We conclude that GSR sensors are capable of capturing stress responses to traffic conflicts and road conditions. There was no correlation or link between normalized GSR data and variables like power, energy, speed, or temperature; i.e., the normalization removed any potential explanatory effect of the variables that may affect sweating.

By matching video and GSR data, it is possible to observe that most of the subjects felt stressed while biking next to traffic, when the bike trajectory was blocked by cars or people, at intersections, and when the bikeway narrow or rough. Cyclists have been found to be statistically more comfortable in physically separated bicycle infrastructure than in other facilities. Peak traffic conditions and intersections also have a significant impact 
on cyclists' stress levels. The results of the research strongly suggest that narrow bike lanes or lanes too close to parked vehicles must be avoided. It is better to give cyclists the right to share the road. However, it is important to mention that the speed limit in the Portland downtown area is mostly 20 miles per hour. It is not possible to generalize these results to other cycling environments without a new data collection and analysis.

Overall, qualitative results presented in this research agree with previous research results. For example, previous research found that cyclists' levels of comfort are directly related to proximity to motorized traffic (Kim, Kim, Ulfarsson, \& Portello, 2007) (Chuang, Hsu, Lai, Doong, \& Jeng, 2013) and separated, off-street bicycle paths are preferred by users (Garder, Leden, \& Pulkkinen, 1998) (Winters, Davidson, Kao, \& Teschke, 2011) (Sener, Eluru, \& Bhat, 2009). In Portland, GPS data analysis and user responses show that cyclists' comfort levels significantly drop if automobile and/or commercial vehicle traffic are identified by the cyclist as a source of stress; if the cyclists identify that the source of stress is pedestrian traffic, there is no significant drop in comfort levels (Blanc and Figliozzi, 2016). However, comfort and safety may not be always correlated. Stress may be caused by high levels of alertness even though the situation is not dangerous. For example riding in a shared path with many pedestrians and potential conflicts may not be unsafe for the cyclist but it may be stressful because the cyclist is in a state of high alertness.

A contribution of this study is the quantification of average stress changes by time of the day, intersections, and bicycle facility type. Readers should also be mindful of the limitations of this research. This one of the few research efforts using GSR in the bicycle transportation context, and results are specific to the cycling conditions found in Portland and this route. It is not evident how these results will apply or transfer to other environments and/or urban areas. Future research endeavors should extend the data collection to more facilities, different urban areas, and more cyclists.

A potential application of this research is to map a city network in terms of measured stress levels, as shown in Figure 3. Cyclists will be able to choose different route options based on stress levels rather than distance or time alone. In the future, by better understanding how traffic conditions and roadway design affect stress levels, planners and transportation engineers may be able to implement policies and designs suitable for less confident or more stress-prone riders.

The normalized-data maps and the results of the models are objective in the sense that they come from physical measurements, i.e. they do not include personal or subjective impressions or opinions. On the other hand, a disadvantage of the method utilized in this research is cost and complexity. It is expensive to buy the GSR equipment, smartphone, software and a bicycle that can register power, energy, speed, and other variables second by second. In addition, running the experiments, collecting the data, and analyzing the data are significantly more time consuming than obtaining data from a questionnaire. The objectivity and high level of detail that can be obtained from GSR sensors come at the expense of additional time and monetary resources. Further studies are required to compare GSR data and traditional questionnaires in more cities and with more cycling facilities. Potentially, naturalistic and non-naturalistic approaches can complement each other in order to obtain the best outcome in terms of both accuracy and resource intensity. Lastly, future research can compare stress levels across modes (e.g. walking, cycling, transit, and driving). Future research results may indicate whether on-road stress affects or explains cycling mode choice share.

\section{Acknowledgements}

The authors would like to acknowledge the support of NITC (National Institute for Transportation and Communities) for funding this research effort. Any errors or omissions are the sole responsibility of the authors. 


\section{References}

Ax, A. F., 1953. The phsysiological differentiation between fear and anger in humans. Psychosomatic Medicine, Volume 55, pp. 466-445.

Barcikowski, R., 1981. Statistical Power with Group Mean as the Unit of Analysis. Journal of Educational Statistics, Volume 6, pp. 267-285.

Benedek, M. \& Kaernbach, C., 2010. Decomposition of skin conductance data by means of nonnegative deconvolution. Psychophysiology, pp. 647-658.

Blanc, B. \& Figliozzi, M., 2016. Modeling the Impacts of Facility Type, Trip Characteristics, and Trip Stressors on Cyclists' Comfort Levels Utilizing Crowdsourced Data. Transportation Research Record.

Boucsein, W., 2012. Electrodermal Activity. New York: Springer.

Chuang, K. H.et al., 2013. The use of a quasi-naturalistic riding method to investigate bicyclists' behaviors when motorists pass. Accident Analysis \& Prevention, Volume 56, pp. 32-41.

Dawson, M., Schell, A. \& Filion, D., 2007. Handbook of pschophysiology. s.1.:Cambridge: University Press.

Dozza, M., Werneke, J. \& Fernandez, A., 2012. Piloting the Naturalistic Methodology on Bicycles. Helmond, The Netherlands, Proceedings, International Cycling Safety Conference 2012 .

Ekman, P., Levenson, R. W. \& Friesen, W. V., 1983. Autonomic nervous system activity distinguishes among emotions. Science, Volume 221, pp. 1208-1201.

Fowles, D. et al., 1981. Publication Recommentadionts for Electrodermal Measurements. Psychophysiology. Psychophysiology, pp. 232-239.

Friedlund, A. \& Izard, C., 1983. Electromyographic Studies of Facial Expressions of Emotions and Patterns of Emotions. In: Social Psychophysiology: A Sourcebook,. s.l.:Guilford Press, pp. 243-286.

Garder, P., Leden, L. \& Pulkkinen, U., 1998. Measuring the Safety Effect of Raised Bicycle Crossings Using a New Research Methodology. Transportation Research Record, Volume 1360, pp. 64-70.

Harvey, F., Krizek, K. J. \& Collins, R., 2008. Using GPS data to assess bicycle commuter route choice.

Washington, DC.

Healy, J., 2000. Wearable and Automotive Systems for Affect Recognition from Physiology, Doctoral dissertation, Massachusetts Institute of Technology.

Healy, J. A. \& Picard, R. W., 2005. Detecting Stress During Real-World Driving Tasks Using Physiological Sensors. IEEE Transactions on Intelligent Transportation Systems, 6(2), pp. 156-166.

Helander, M., 1978. Applicability of drivers' electrodermal response to the design of the traffic environment. Journal of Applied Psychology, pp. 481-488.

Hong, J. H., Ramos, J. \& Dey, A. K., 2012. Stress Recognition: A step outside the lab. New York, UbiComp '12 Proceedings of the 2012 ACM Conference on Ubiquitous Computing.

Johnson, M., Newstead, S., Charlton, J. \& Oxley, J., 2001. Riding through red lights: The rate, characteristics and risk factors of non-compliant urban commuter cyclists. Accident Analysis \& Prevention, 43(1), pp. 323-328.

Jones, T., Chatterjee, K., Spinney, J., Street, E., Van Reekum, C., Spencer, B., Jones, H., Leyland, L.A., Mann, C., Williams, S. \& Beale, N., 2016. cycle BOOM. Design for Lifelong Health and Wellbeing. Summary of Key Findings and Recommendations. Oxford Brookes University, UK. http://cycleboom.org/wpcontent/uploads/2016/09/cB_Summary_Report_Sept2016_Digital.pdf. Accessed June 1, 2017.

Kahneman, D., 1973. Arousal and attention. Attention and Effort. Englewood Cliffs: Prentice-Hall, pp. $28-49$.

Kim, J., Kim, S., Ulfarsson, G. \& Portello, L., 2007. Bicyclist injury severities in bicycle- motor vehicle accidents. Accident Analysis \& Prevention, pp. 238-251. 
Labbé, E., Schmidt, N. \& Babin, J., 2007. Coping with Stress: The Effectiveness of Different Types of Music. Appl Psychophysiol Biofeedback, Volume 32, pp. 163-168.

Lanzetta, J. \& Orr, S., 1986. Excitatory Strength of expressive faces: effects of happy and fear expressions and context on the extintion of a conditioned fear response. Journal of Personality and Social Psychology, Volume 50, pp. 190-194.

Levenson, D. \& Edelberg, R., 1985. Scoring criteria for response latency and habituation in electrodermal research: a critique. Psychophysiology, pp. 417-426.

Lisetti, C. \& Nasoz, F., 2004. Using Noninvasive Wearable Computers to Recognize Human Emotions from physiological signals. EURASIP Journal on Applied Signal Processing, pp. 1672-1687.

Mekuria, M., Furth, P. \& Nixon, H., 2012. Low-Stress Bicycling and Network Connectivity. MTI Report 11-19, San Jose,CA.: MINETA Transportation Institute.

Pecchinenda, A. \& Smith, C., 1996. Affective signigicance of skin conductance activity during difficult problemsolving task. Cognition and Emotion, pp. 481-503.

Picard, R., Vyzas, E. \& Healy, J., 2001. Toward Machine Emotional Intelligence: Analysis of Affective Physiological State. IEEE, pp. 1175-1191.

Prokasy, W. \& Raskin, D., 1973. Electrodermal activity in psychological research. New York: Academic Press, INC.

Sener, I. N., Eluru, N. \& Bhat, C. R., 2009. An analysis of bicycle route choice preferences in Texas, US. Transportation, 36(5), pp. 511-539.

Seyle, H., 1956. The Stress of Life. New York: McGraw-Hill.

Sharma, N. \& Gedeon, T., 2012. Objective measures, sensors and computational techniques for stress recognition and classification: A survey. Computer Methods and Programs in Biomedicine, pp. 1287-1301.

Sorton, A. \& Walsh, T., 1994. Bicycle Stress Level as a Tool to Evaliate Urban and Suburban Bicycle Compatibility. Transportation Research Record, Volume 1438, pp. 17-24.

Transportation Research Board, 2010. Highway Capacity Manual 2010. Washington, DC: Transportation Research Board.

Weihong, Z., Zhu, Z. \& Quiang Ji, 2005. A Real-Time Human Stress Monitoring System Using Dynamic Bayesian Network.

Wilfrid, J. \& McLachlan, M., 1992. Characteristics of function-specific pathways in the sympathetic nervous system. Perspectives, 15(12), pp. 475-481.

Winters, M., Davidson, G., Kao, D. \& Teschke, K., 2011. Motivators and deterrents of bicycling: comparing influences on decisions to ride. Transportation, 38(1), pp. 153-168. 